\title{
The role of the saccade target object in the perception of a visually stable world
}

\author{
CHRISTOPHER B. CURRIE and GEORGE W. MCCONKIE \\ University of Illinois, Urbana-Champaign, Illinois \\ LAURA A. CARLSON-RADVANSKY \\ University of Notre Dame, Notre Dame, Indiana \\ and \\ DAVID E. IRWIN \\ University of Illinois, Urbana-Champaign, Illinois
}

\begin{abstract}
Although the proximal stimulus shifts position on our retinae with each saccade, we perceive our world as stable and continuous. Most theories of visual stability implicitly assume a mechanism that spatially adjusts perceived locations associated with the retinal array by using, as a parameter, extraretinal eye position information, a signal that encodes the size and direction of the saccade. The results from the experiment reported in this article challenge this idea. During a participant's saccade to a target object, one of the following was displaced: the entire scene, the target object, or the background behind the target object. Participants detected the displacement of the target object twice as frequently as the displacement of the entire background. The direction of displacement relative to the saccade also affected detectability. We use a new theory, the saccade target theory (McConkie \& Currie, 1996), to interpret these results. This theory proposes that retinal (as opposed to extra-retinal) factors, primarily those concerning the saccade target object, are critical for the detection of intrasaccadic stimulus shifts.
\end{abstract}

How we "see" or perceive the world is not strictly determined by our retinal image. The constant, stable environmental structure that we perceive must be derived from a sensory input stream that is noisy and discontinuous. For example, if the world or parts of it move sufficiently during a fixation, we perceive displacement and motion. However, when the light pattern on the retina moves because of a saccade, we do not perceive motion or displacement; rather, we perceive stability (traditionally known as space constancy). How does this happen?

In explaining stability, researchers have addressed two issues: (1) why retinal motion is not perceived as motion in the world during a saccade and (2) why the displacement of the light pattern on the retina is not perceived as displacement in the world from one fixation to the next. Researchers have made great gains in understanding the mechanisms involved in the suppression of a perception

We thank Bruce Bridgeman, Dan Simons, and an anonymous reviewer for their insights, comments, and helpful suggestions, Sheila Daniels for creating Figure 1, and last but not least, Gary Wolverton for developing the data collection, eye-movement contingent display change, and data reduction software used in this experiment. At the time of data collection, all the authors were affiliated with the Beckman Institute for Advanced Science and Technology at the University of Illinois in Urbana-Champaign. Correspondence concerning this article should be addressed to C. B. Currie, Center for Cognitive Science and Laboratory of Vision Research, Psychology Addition-Busch Campus, Rutgers University, 152 Frelinghuysen Road, Piscataway, NJ 08854-8020 (e-mail: fantasticks@netscape.net). of motion during a saccade. Specifically, when the image of the world sweeps across the retina during a saccade, velocities of up to hundreds of degrees/second can be reached. Because the retinae cannot successfully transduce a high-spatial-frequency component of the proximal stimulus at this speed, any perception of such a component shifting across the retinae is physically impossible (Grusser, 1972). At the same time, selective suppression of the magnocellular visual pathway suppresses the resultant fast, low-spatial-frequency retinal image blur that accompanies the saccade (Burr, Morrone, \& Ross, 1994). Finally, visual masking from the postsaccade fixation suppresses any perception of a smeared but structured retinal image that is present at lower velocities near the end of the saccade (Campbell \& Wurtz, 1978; Grusser, 1972; Volkmann, Schick, \& Riggs, 1968). Unfortunately, we do not have a comparable understanding of why the displacement of the light pattern on the retina after a saccade is not perceived as displacement in the world. Theories concerning this issue can be divided into two groups. We will refer to these two groups as extra-retinal and target object theories.

\section{EXTRA-RETINAL THEORIES}

Extra-retinal theories rest on the assumption (which goes back to Helmholtz, 1866/1963) of the existence of a visual, neurosensory pathway that maps retinal locations to unique corresponding positions in a neural map that 
represents perceived positions in the world (see, e.g., von Holst \& Mittelstaedt, 1971). With this assumption, eye movements introduce a "problem" for visual stability.

Extra-retinal-based theories propose that, in order to see the world as stable, the system encodes the size and direction of the saccade in a neural signal. The system then uses the size and direction encoded in this neural signal as parameters to remap all retinal locations to new perceived positions (Bridgeman, van der Heijden, \& Velichkovsky, 1994; Matin, 1986). Researchers in the field have commonly referred to this neural signal as extra-retinal eye position information (or EEPI, after Matin, 1986).

A further (albeit, implicit) assumption is that the remapping occurs over the entire retinal array (a global assumption) and that the remapping occurs automatically with each saccade. For example, several neurons in the lateral intraparietal area have been shown to exhibit retinotopic remapping of their receptive fields before a saccade is made (Duhamel, Colby, \& Goldberg, 1992). This remapping could support stability by contributing to the integration of information across saccades. A critical finding from Duhamel et al. is that this retinotopic remapping does not happen with shifts of attention to the saccade target when a saccade is not made. This suggests that visual stability could be the result of a low-level, automatic mechanism that operates independent of attention. In addition, Haarmeier, Thier, Repnow, and Petersen (1997) report the case of a patient with bilateral extrastriate cortex lesions that damaged a portion of the parieto-occipital region. This region may discriminate between self-induced and externally induced visual motion signals by comparing them with a reference signal encoding an eye movement. This patient suffered from false perception of motion of the world whenever he engaged in self-induced smooth-pursuit eye movements.

In sum, these studies all suggest that a low-level mechanism may operate to stabilize perception by taking into account information from many retinal locations ( $\mathrm{Du}-$ hamel et al., 1992).

A strict prediction of extra-retinal theories is that any mismatch between the EEPI signal and the postsaccadic retinal signal will be attributed to a change in the world. Researchers have used studies of intrasaccadic stimulus shifts to examine the sensitivity and accuracy of proposed EEPI-based mechanisms. In these studies, a physical stimulus is displaced during a viewer's saccade so that its position in the world is different from one fixation to the next. The viewer's task in these studies is usually to detect any displacement of the stimulus. Contrary to the strict prediction that any change will be detected, stimulus shifts during saccades often escape detection. A common finding has been that the likelihood of detecting a stimulus shift of a given size decreases as saccade length increases (Bridgeman, Hendry, \& Stark, 1975; Li \& Matin, 1990; Mack, 1970; McConkie \& Currie, 1996; Whipple \& Wallach, 1978).

The typical interpretation of these detection failures is that the extra-retinal information concerning eye po- sition is imprecise or noisy and that the noise or amount of imprecision increases with saccade length (Li \& Matin, 1990). Because of this, many researchers have concluded that an extra-retinal-based compensation theory is an incomplete account of visual stability (Macknik, Fisher, \& Bridgeman, 1991; Skavenski, 1990).

If this is so, the perceptual system must employ a mechanism to detect any mismatch between the magnitude of a retinal image shift predicted by the EEPI signal and the magnitude of an actual retinal image shift. The problem then becomes how to determine how much of the mismatch is due to error in the system and how much is due to displacement in the world. One possibility is a mechanism that sets a threshold. For example, if the mismatch is below the threshold, the system suppresses a perception of displacement; if it is above the threshold, an observer will perceive displacement (Li \& Matin, 1990).

\section{TARGET OBJECT THEORIES}

As the basis for explaining the failure to detect stimulus displacements accompanying saccades, another branch of research has focused on the role of retinal, ${ }^{1}$ (as opposed to extra-retinal) factors. This line of research has challenged several assumptions of EEPI-based theories. For example, Bridgeman (1981) and Brune and Lücking (1969) observed that when a picture was shifted during a saccade, rather than perceiving the entire picture to shift, participants indicated that only the objects to which they were sending their eyes seem to shift. These results suggest that local stimulus information near the landing site of the eyes is critical for the perception of stimulus displacement. These studies leave open the question of exactly how this information is used to stabilize perception.

McConkie and Currie (1996) also found evidence for local, as opposed to global, processing of a shifted retinal image. In one experiment, while participants freely viewed a full-color, natural scene, the scene was shifted during preselected saccades. For a picture shift of a constant size, they found an inverse relationship between the likelihood of detection and the length of a saccade. Such a result is consistent with the idea of a noise level in the extra-retinal signal that increases with saccade length ( $\mathrm{Li}$ \& Matin, 1990).

At the same time, McConkie and Currie (1996) obtained a result that was not accounted for by the current versions of EEPI-based theories. First, the direction of the saccade relative to the direction of the shift of the picture influenced the frequency of detecting shifts. Specifically, shifts in the same direction as that of the saccade were detected more often than shifts in the opposite direction. McConkie and Currie explained this by noting that the eyes tend to undershoot their target objects in scenes (Henderson, 1993). Shifting the picture in the opposite direction to that of the saccade results in the eyes' landing closer to their target then when the picture is shifted in the same direction as that of the saccade. Thus, 
detection of a shift is lower in the condition in which the eyes land closer to the target object. Macknik et al. (1991) also found that shift direction relative to saccade direction influenced detection, but their results were the opposite of those obtained by McConkie and Currie. This effect, then, appears to be local in nature, which is compatible with target object theories but not with current versions of EEPI-based models.

In sum, EEPI-based theories propose that an extraretinal signal is used to update, with each saccade, the perceived visual directions of all objects currently in view. Another line of research has focused on retinal factors-specifically, those located in the region of a saccade target object. This theory, in line with O'Regan's (1992) view, suggests that the visual system assumes a stable world unless there is evidence to the contrary. The saccade target object may play a unique role in the detection of intrasaccadic stimulus shifts.

\section{The Saccade Target Object Theory}

Although results supporting target object theories do not rule out the involvement of an extra-retinal signal, they do indicate a need for a revision in EEPI-based theories. Such a revision would need to give priority to the remapping of local information near the landing site of the eyes. As an alternative, however, we have proposed a localist, retinal-based theory of visual stability, called the saccade target object theory (McConkie, 1991; McConkie \& Currie, 1996). This theory does not assume that the system must somehow correct for the problem of a shifting retinal image in order to produce a perception of a stable world. Rather, the theory assumes that visual stability arises as a by-product of the successful application of several processing operations that are in the service of a fundamental perceptual goal: the selection and identification of a saccade target object. The theory proposes that these processes occur at the beginning of each new fixation because they are required before any further analysis of a scene can take place.

Specifically (see McConkie \& Currie, 1996, for more details), the theory assumes that each saccade is normally an attempt to direct the center of vision toward some entity, which we call the saccade target object. Once the system selects the saccade target, its position in the mental representation of the scene is noted.

At this point, eye movements do introduce a "problem" for the perceptual system. Because there is no evidence that there is a carryover of a veridical, visual representation of the retinal array (Irwin, 1992b), the system faces a fundamental perceptual goal of "finding" the saccade target object at the beginning of each new fixation. The theory assumes that near the end of each fixation, the system selects and temporarily stores critical locating features about the selected target object to facilitate a search process when information from the new fixation begins to become available. The nature of these features is an open question. The theory assumes that they are, most likely, visually based, but their nature could vary by task, stimulus, or eccentricity of the saccade target. Furthermore, the theory assumes that a shift of visual attention before a saccade facilitates both the encoding of these features and their retention across a saccade (Irwin, 1992a).

At the onset of the new fixation, the critical locating features are used to find the saccade target object within a limited retinal search region, probably near or at central vision.

When the saccade target is found in the stimulus array, the system can identify the relationship between its retinal location and its location in the mental representation that the system noted before the saccade. Once these two corresponding points of reference are established, a bidirectional mapping exists between the contents of the representation of the scene and the retinotopic representation of the scene currently in view. With this function, identifying an object or location in one space gives immediate access to the corresponding object or location in the other space.

We propose that intrasaccadic stimulus shifts disrupt these target-locating processes by reducing the likelihood that the saccade target object will lie within the limited retinal search region. When this happens, the system initiates an error correction process, and the viewer experiences this as a lack of perceptual stability.

Unfortunately, the involvement of a saccade target object in the studies reviewed above is inferred, rather than being established by direct evidence. This is because these studies allowed free viewing of the stimulus, and thus, it was not possible to identify the object to which subjects were sending their eyes on a given saccade. Moreover, they shifted the entire stimulus. Thus, it is not possible to unconfound the effects of a local shift (i.e., the saccade target object) from those of a global shift (i.e., the entire retinal image). The primary goal of the experiment reported in this article was to test specifically for the role of a saccade target object in the detection of intrasaccadic stimulus shifts.

Briefly, in the present experiment, we had participants move their eyes to a predefined target object in a natural scene (see Figure 1). During the saccade to the target object, the entire picture shifted (all shift), just the target object shifted (object shift), the entire picture except for the target object shifted (background shift), or there was no shift (control). The task of a participant was to detect any change in the picture.

We hypothesized two basic possible patterns of results. (1) If there is a global remapping of the retinal image into a stable spatiotopic store from one fixation to the next, the more features in the picture that are shifted, the more detectable the shift should be. Thus, the detection rate would be highest for the all shift condition, in which the entire picture changed location. Detection should be next highest for the background shift condition, in which all but the saccade target changed location. Detection should be least for the object shift condition, in which only the target object moved. Such a result 


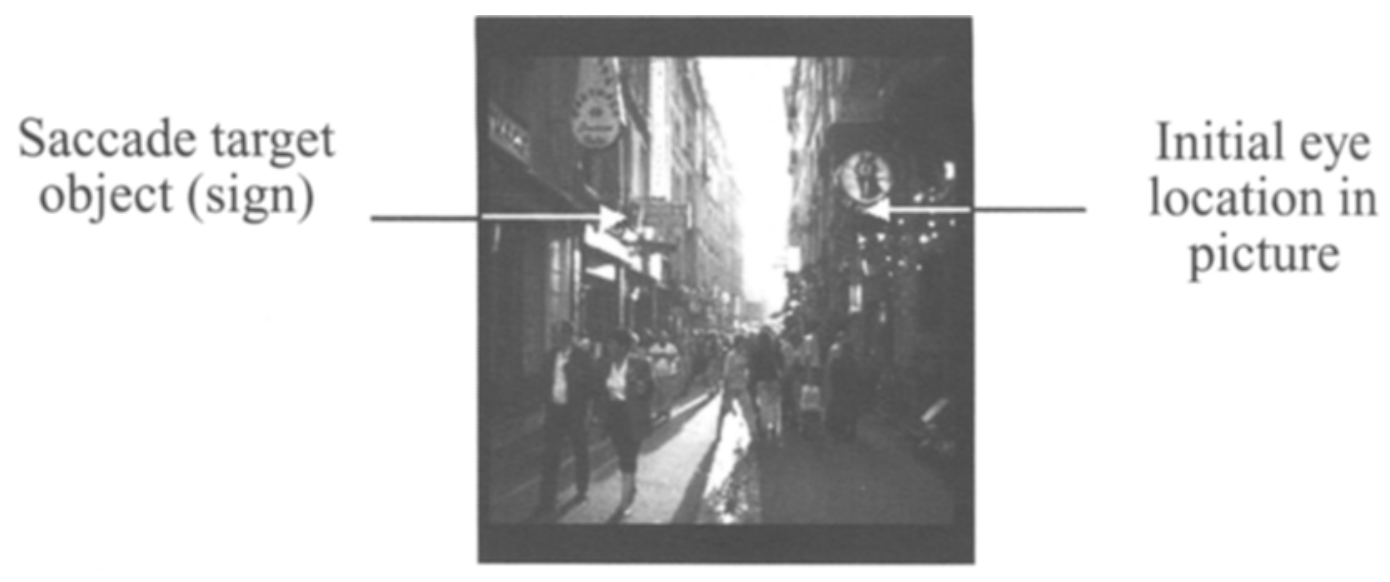

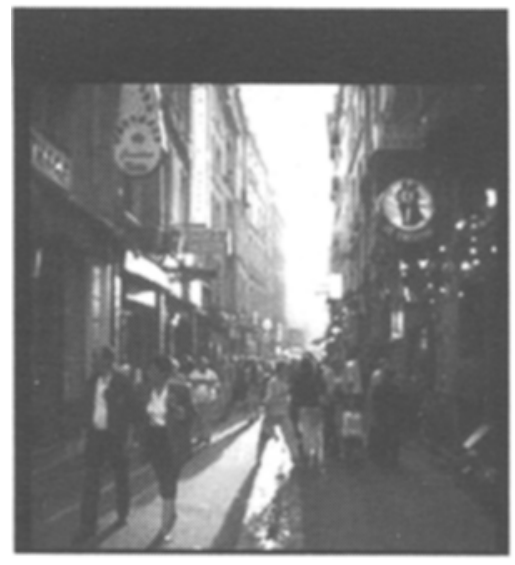

All shift down

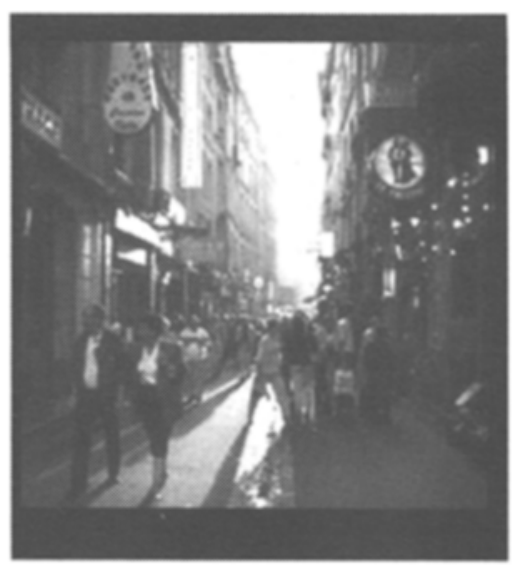

Object shift down

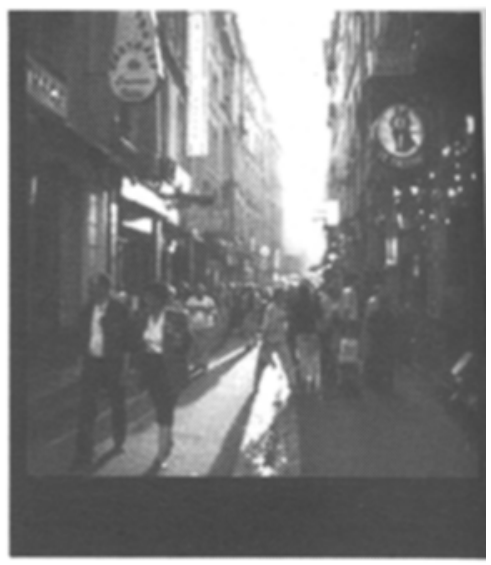

Background shift up

Figure 1. Sequence of stimulus events. The participants first fixated an initial fixation box and then pressed a button that caused a $1^{\circ} \times 1^{\circ}$ star to appear $2^{\circ}$ in their periphery (not shown in the figure). Top: During the saccade to the star, the star was removed, and a full-color, 16-bit, digitized magazine picture appeared. Bottom: During the saccade to the target object and before the end of that saccade, one of three experimental manipulations occurred. All the shift distances were $2^{\circ}$ right or left, up or down. These frames in this figure show an example of an all shift down, object shift down, and background shift up. There was also a control condition in which the picture did not shift, but this is not shown in the figure.

would be compatible with the global assumption of EEPIbased theories. (2) If only the saccade target object is used to detect intrasaccadic stimulus shifts, the all shift and object shift conditions should be equally detectable. Detections of the background shift, on the other hand, should be close to the frequency of false positives in the control condition.

\section{METHOD}

\section{Participants}

Eleven individuals from the University of Illinois community participated as paid $(\$ 5 / \mathrm{h})$ participants. All had 20/20 uncorrected vision.

\section{Apparatus}

The pictures were displayed on a Princeton Ultra-Synch monitor in $756 \times 486$ pixel mode, controlled by an ATVista display con- troller card in a 386 computer and refreshed at $60 \mathrm{~Hz}$. In order to hide the edge of the display screen, the plastic molding of the monitor was blackened, and a large $41^{\circ} \times 40^{\circ}$ glare filter was placed in front, making it difficult to see the borders of the computer display or its case. In the ATVista display controller, four images were stored in its image memory. This made it possible to switch from one image to another simply by switching to a different region in image memory. Such a change could occur at the end of any horizontal refresh scan; thus, the computer could initiate a switch at any point during a vertical refresh of the screen, and it would be completed in $16.7 \mathrm{msec}$.

The observer's eyes were tracked with a fifth generation Dual Purkinje Image Eyetracker that sampled eye position every millisecond and produced 12-bit data values for horizontal and vertical positions. The equipment was adjusted so that a $1^{\circ}$ movement produced a 200 value change in the eyetrack signal. Saccades were identified within $10 \mathrm{msec}$ of their initiation, and on the onset of a predefined critical saccade, an image change was initiated immediately. Saccade onsets were detected on line as a difference of 
10 values or more (i.e., 3 min of arc) between the current sample and one taken $4 \mathrm{msec}$ earlier, followed by four additional samples indicating movement in the same direction. Thus, the saccadic detection threshold was movement between $12^{\circ}$ and $15^{\circ} / \mathrm{sec}$ (depending on the spatial resolution of the eye tracker during calibration). Since image change could begin at any point during a vertical refresh cycle, the display of the old image was discontinued no later than $10 \mathrm{msec}$ after the onset of a saccade. The new image completely overwrote the old within $16 \mathrm{msec}$.

\section{Design and Procedure}

Figure 1 shows the sequence of events in each type of trial. We used six pictures, approximately $13.6^{\circ}-16.2^{\circ}$ horizontal $\times 11.0^{\circ}-11.5^{\circ}$ vertical. Five pictures were outdoor scenes; one was an indoor scene. Each trial began by having the participant direct his or her gaze to a fixation point on an otherwise blank screen. A small star appeared $2^{\circ}$ in their periphery, to which the participant was instructed to shift his or her gaze. During the saccade to this star, it disappeared, and a full-color, natural scene appeared (top picture in Figure 1). The purpose of the star was, first, to avoid a sudden onset of a picture during a fixation, which could trigger an automatic saccade, and second, to control for eye position relative to a saccade target object. One hundred milliseconds into this first fixation on the picture, one object in the picture (e.g., the small, square sign below the tall "La Petite Taverne" vertical marquee in Figure 1) instantaneously moved $1 / 5^{\circ}$ and then returned to its original position $32 \mathrm{msec}$ later; this signaled to the participant which object to look at (Boyce \& Pollatsek, 1992). The distance of the eyes to the center of the target object was always $6^{\circ}$. Since the distance to the target object was fixated at $6^{\circ}$, we did not want the participant to see the wiggled object from the same position in the scene each time it was presented. In order to vary location of the eyes relative to the target object, the star was located at some point on an imaginary circle with a radius of $6^{\circ}$ centered on the target object. Imagining a horizontal line running through the center of this circle, the star, or starting point of eyes, was $0^{\circ}, 20^{\circ}, 40^{\circ}$, or $60^{\circ}$ of arc above or below this line. In half the pictures, the eyes approached the target object from the right; in the other half, the eyes approached from the left. Because these were natural pictures, it was not possible to have all possible starting points in each picture. For example, a target object may have been located relatively near the top of a particular picture. In that case, it was more natural to have the eyes approach the object from below. Thus, in four of the pictures, the starting point was above the imaginary horizontal line, and in two of the pictures, the starting point was below the imaginary horizontal line.

During the saccade to the target object, one of four types of changes occurred in the scene: (1) a control condition with no change; (2) an all shift condition with a change in the position of the entire scene; (3) an object shift condition, in which only the target object shifted, with the background remaining stable; (4) a background shift condition, in which the target remained at the same location on the screen but the rest of the scene shifted. All the shifts were $2^{\circ}$, about $1 / 3$ of the saccade length. Three pictures shifted right and left an equal number of times; the other three shifted up and down an equal number of times. The participants then reported whether or not they noticed any change in the picture by pressing a button. The picture remained on until the participant responded. The size of the target object ranged from an average of $2.5^{\circ} \times 2.5^{\circ}$ to $0.7^{\circ} \times 0.7^{\circ}$, and each was selected so that it could change position by the required amount without violating normal, real-world spatial constraints.

Six pictures each had four starting points (star locations) and four picture shift conditions (all, background, object, and control), for 96 combinations. Each participant experienced each combination twice, resulting in 192 trials per participant. The pictures and conditions were presented in random order.

\section{RESULTS}

We excluded 1 participant from the analysis because of an inability to distinguish between the control condition and the other shift conditions. This left 10 subjects. In addition, we excluded one stimulus picture because the participants could not detect the $1 / 5^{\circ}$ shift that signaled the target object location. Of the remaining 1,600 cases, we discarded 280 cases because of eye-tracking loss of lock. We discarded 51 more cases because saccades to the star resulted in the eyes' landing more than $2^{\circ}$ from its center. Finally, we excluded an additional 139 cases because saccades to the preshift target object (see the top panel, Figure 1) resulted in the eyes' landing more than $2.5^{\circ}$ from its center. Thus, about $29 \%$ of the potentially analyzable data were lost, and the final data set contained 1,136 cases.

\section{Likelihood of Detection}

Figure 2 shows the frequency of detecting a shift in each of the four different shift conditions. A repeated measures, general linear analysis, using 10 subjects and shift condition as a factor, showed a significant effect $[F(3,27)=$ $68.76, p<.001$ ]. Single degree comparisons (using $M S_{\mathrm{e}}=$ 0.053 from the general linear analysis) were used to test for differences in detection frequencies between the shift conditions as described by the above hypotheses. First, a comparison between the object shift and the all shift conditions ( $83 \%$ and $74 \%$ detection rates, respectively) failed to reach significance $[F(1,27)=2.22]$. Second, a comparison between the average of the object and all shift conditions $(78.5 \%)$ and the background shift condition $(34 \%)$ revealed that shifts involving the saccade target object were more detectable than shifts involving the background alone $[F(1,27)=67.10, p<.001]$. Third, the difference between the object and the background shift conditions was also significant $[F(1,27)=61.46, p<$ $.001]$. Finally, a comparison between the background shift condition and the control/false alarm condition $(4 \%$ detection) revealed that a shift of the background resulted in detections that were significantly above the false alarm rate $[F(1,27)=23.29, p<.002]$.

The observation that shifting only the saccade target (object shift condition) is more detectable than holding the saccade target stable and shifting everything else in the picture (background shift condition) supports the idea that visual stability depends primarily on local retinal information concerning the saccade target object. The same can be said for the observation that shifting the target object alone (object shift condition) is as detectable as shifting the entire picture (all shift condition).

Because increasing saccade length while holding the magnitude of a stimulus shift constant is known to decrease detection (Bridgeman et al., 1975; Li \& Matin, 1990; Mack, 1970; McConkie \& Currie, 1996), it is important to know whether saccade length is constant in each 


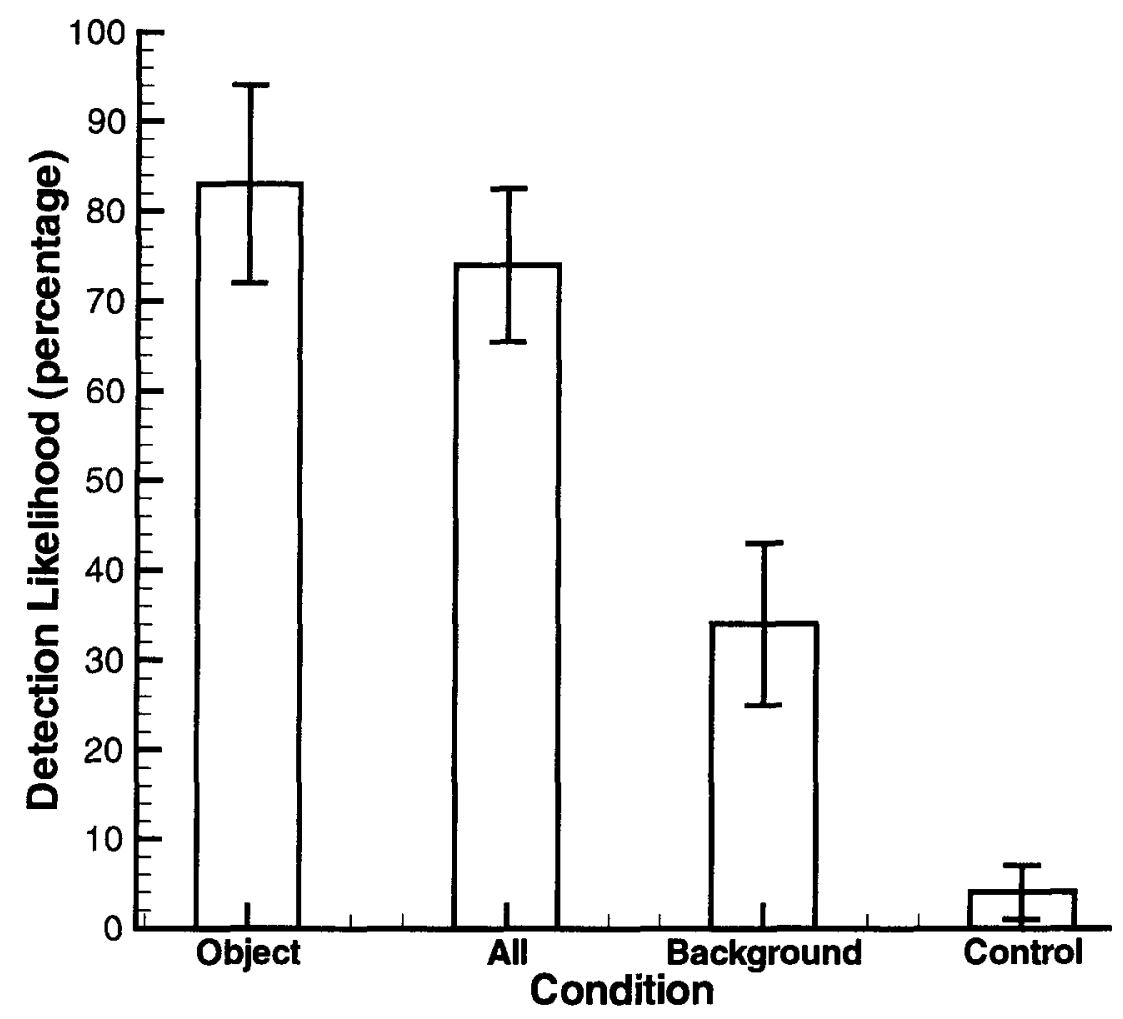

Figure 2. Likelihood of detection in the four shift conditions. Error bars are standard deviations of the mean.

shift condition. Using shift condition as a factor and preview distance (i.e., as measured from the eye's location at the star to the center of the preshift target object) as a dependent measure, a repeated measures, general linear model analysis yielded no effect of shift condition on preview distance $[F(3,9)=1.0]$. Specifically, in each condition, the average preview distance was $6^{\circ}$. We also looked at oculomotor accuracy in saccading to the target object. That is, we used the distance between the landing site of the eyes after the saccade to the target object and the center of the preshift target object as a dependent measure and shift condition as a factor in a repeated measures, general linear model analysis and found no effect of shift condition on accuracy $[F(3,9)=0.82]$. Specifically, the eyes, on average, undershot the center of the preshift target object in each shift condition by about $0.5^{\circ}$. Since the participants were equally accurate at finding and saccading to the target object in each shift condition, differences in the detection likelihoods in Figure 2 cannot be explained by differences in actual saccade length or accuracy.

Another concern is the possibility that repeating each scene multiple times, with the same target object, could have led to additional encoding of the target objects. This could have produced the higher detection of their displacement in the all and object shift conditions. This concern was addressed by looking at the detection likelihoods for those trials in which the pictures were presented for the first two times ${ }^{2}$ (early view) and those in which the pictures were presented at all subsequent times (later view). If picture repetition is responsible for the main effect presented above, trials in which the picture is presented only for the first two times should not show the pattern of data shown in Figure 2.

Figure 3 shows the early view and later view data. It appears that detection levels may increase slightly with picture repetition, but the pattern of detection rates across the four shift conditions is similar to that in Figure 2. The interaction between shift condition and picture repetition failed to reach significance: $[F(3,24)=0.68]$. Thus, there was little difference in the overall pattern of detection between the early view of a picture and later views.

\section{Response Time and Multiple Bases for Detection}

The significantly higher detection rate in the object and all conditions over the background condition does not support the idea of a mechanism that remaps the visual direction of all the objects in the scene. On the other hand, the observation that the background detection rate was higher than the control (i.e., false alarm) rate would not be expected on the basis of the saccade target theory. In both conditions, the target-error distance is the same. We assume that the detection of the stimulus change in the background condition is based on a different mechanism: the detection of a change in the spatial relationships between the target object and other contextual objects (probably those that are closest to it). However, we further assume that the system cannot detect changes in spatial relations until after the processes involved in establishing 


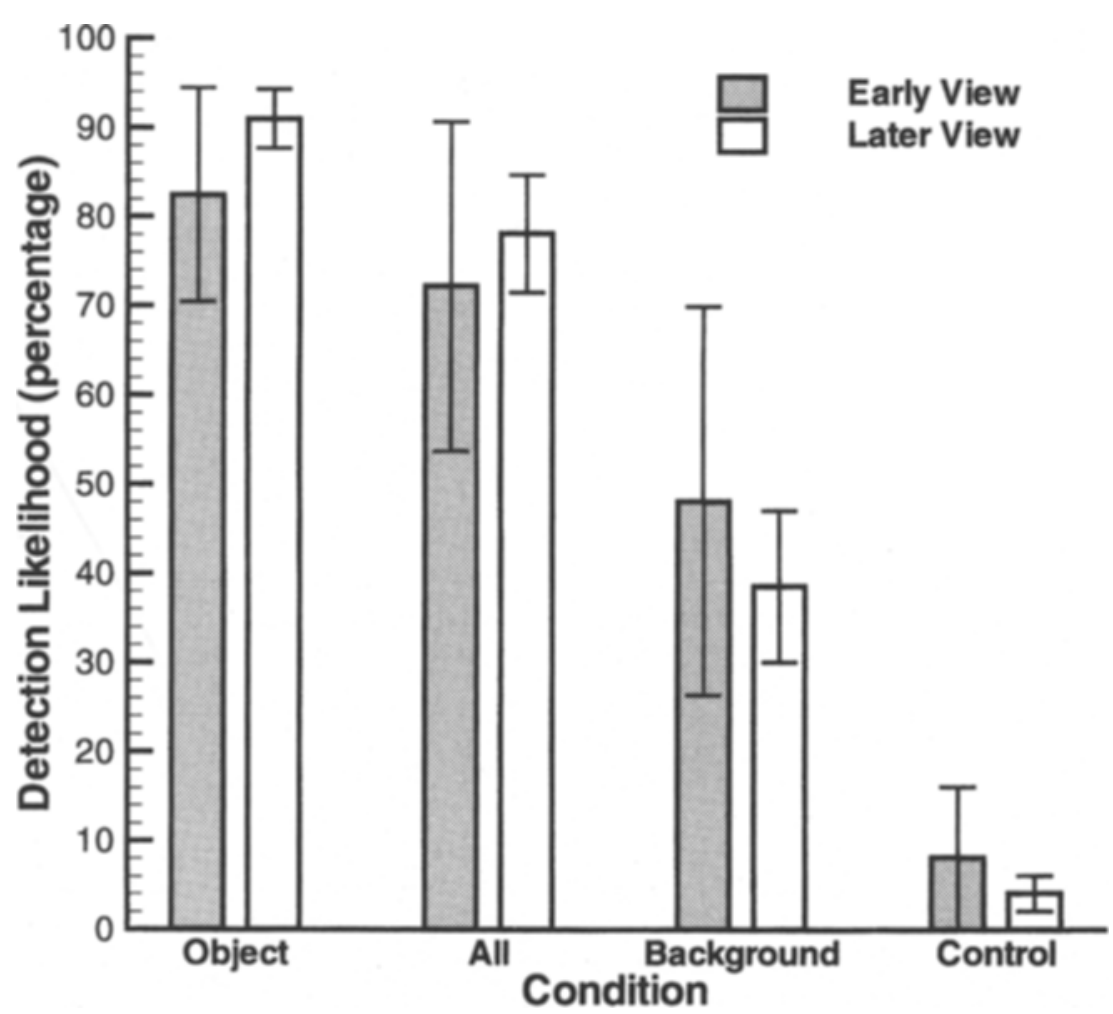

Figure 3. Likelihood of detection for early view verses later view. Error bars are standard deviations of the mean.

the mapping function between the retina and the mental representation are complete. This is because we assume that it involves a comparison between current retinal information and previous represented information. We refer to this as the late spatial relations detection assumption.

With this assumption, there are two bases of detection. If the saccade target is displaced, as in the all and object shift conditions, an early detection is likely as a result of initially failing to locate the target object. If spatial relationships change, as in the background and object shift conditions, the system may detect, at a later point in time, the spatial relation differences between the currently viewed scene and a previously formed mental representation. This leads to two predictions for the present data. First, since the system can detect shifts in the object condition on either basis, this theory predicts a higher detection rate than in any of the other experimental conditions. We observed this pattern in Figure 2, although the difference between the object shift and the all shift conditions was not statistically significant.

Second, if detection is occurring from an early process in some conditions (i.e., object and all, in which the target object is shifted) and from a later process in some conditions (i.e., background and object, in which the spatial relations are changing), the theory predicts differences among the conditions in terms of response times. Specifically, detections should be faster in the all shift condition than in the background shift condition. The object shift condition, which includes detections of both types of changes, should be in between. However, the response times will probably be closer to the all shift condition, since the frequency of detecting from the early process alone is so high.

A repeated measures, general linear analysis, using 10 subjects and three factors, was carried out, and single degree comparisons (using $M S_{\mathrm{e}}=80,335$ ) were used to test for predicted differences among the three experimental shift conditions. A contrast between the averages of the object $(1,088 \mathrm{msec})$ and all $(1,067 \mathrm{msec})$ shift conditions (i.e., $1,078 \mathrm{msec}$ ) and the background shift condition $(1,291 \mathrm{msec})$ revealed a marginally faster response time for the object and all shift conditions than for the background shift condition $[F(1,18)=3.77, p=.0679]$. However, there was no significant difference between background shift and all shift response times $[F(1,18)=$ 3.11]. Thus, the response time data are ordered exactly as would be predicted by the early versus the late detection hypothesis, but the differences between the means were not significant. Also, an examination of the cumulative frequency curves for the response times indicates that the curve for the all shift condition begins to rise $100 \mathrm{msec}$ earlier than that for the background shift condition (600 vs. $700 \mathrm{msec}$ ).

\section{Effect of Target-Error Distance on Detection Likelihood}

The saccade target theory predicts that if saccade length is held constant, the distance between the fixation 
location (or landing site) after a saccade to the target object and the location of the postshift target object (which we call the target-error distance) should play a role in detecting a shift of the saccade target object. Specifically, when the eyes land further from the target object because of a stimulus shift, it is less likely that the target-locating processes will find the saccade target within the limited retinal search region, thus leading to an increased likelihood of detection.

The all shift condition presents the cleanest case by which to test this hypothesis, because we assume that detection in this condition is due to an initial failure to locate the target object within the limited retinal research region, and not to other factors that we assume are operating in the object shift condition, such as detection of changed spatial relations. An analysis of eye landing position relative to the location of the preshift target in the all shift condition showed that the participants, on average, tended to undershoot this location by about $0.5^{\circ}$. This being the case, an all picture shift in the same direction as the saccade should, on average, exacerbate any undershoot and increase the distance between the landing site of the eyes and the postshift saccade target object location. On the other hand, a shift in the direction opposite the saccade should reduce the target-error distance. The data showed the following pattern ${ }^{3}$ : In the same di- rection case, the target-error distance was $2.31^{\circ}$; in the opposite direction case, it was only $1.71^{\circ}$.

A repeated measures, general linear model, using frequency of detection as a dependent measure, supported this target-error distance hypothesis by showing that an all shift in the same direction as the saccade had a significantly higher detection rate than an all shift in the opposite direction [same, $84 \%$; opposite, $53 \% ; F(1,9)=$ $28.75, p<.005]$. Although this result is compatible with the saccade target theory, it is not compatible with current EEPI-based theories.

The role of target-error distance in the object shift condition is more complex because there are two possible bases of detection in this condition: target object displacement and changed spatial relations. Both the all and background shift conditions have a single basis for detection: target object displacement in the all shift condition, and changed spatial relations in the background shift condition. The question arises: How do these two factors combine to produce a perception of target object shift in the object shift condition?

Figure 4 shows the probability of detection, averaged over participants, as a function of postshift target-error distance in each of the shift conditions. In the case of a background shift, the postshift and preshift target locations are the same. In all cases, the distances are absolute

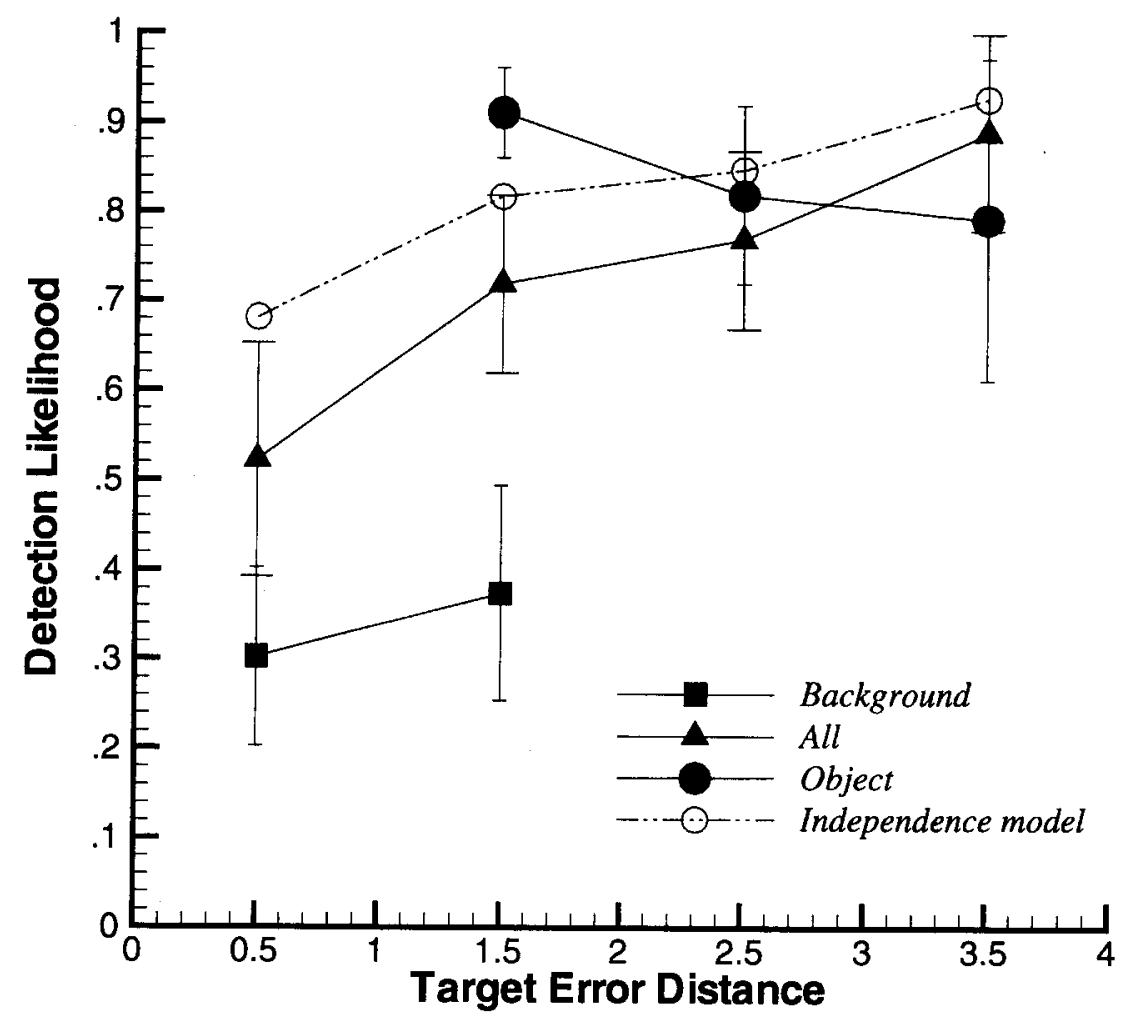

Figure 4. Likelihood of detection as a function of target-error distance for the independence model of object data and the observed data from the background, all, and object shift conditions. Error bars are standard deviations of the mean. 
measures, in the sense that the distinction between overand undershoots is ignored and all the distances are positive numbers. The object, all, and background shift curves were constructed by recording a target-error distance for each trial along with its detection response, either 0 (no detect) or 1. For each participant, the data were sorted on the basis of target-error distance. Bin widths of $1^{\circ}$ (of target-error distance) were defined, and a probability of detection was calculated for each bin. These probabilities were averaged across subjects, to produce the data points in Figure 4.

Analyses of trend were carried out for the curves. A significant linear component was found in the all shift curve $[F(1,26)=20.85, p<.01]$, the condition allowing detection based only on the early processes. This confirms the prediction from the saccade target theory of an increase in detection with an increase in target-error distance. A significant linear trend was also found in the object shift curve $[F(1,15)=4.68, p<.05]$, but in the opposite direction. The slight rise with target-error distance in the background shift condition was not significant $[F(1,9)=0.8]$.

As can be seen in Figure 4, the object shift curve begins above the all shift curve, but the two curves converge at target-error distances above $2.0^{\circ}$. This suggests that the effect of changed spatial relations facilitates detection only when the eyes are near the target object. Comparing curves for the object and the all shift conditions indicates a significant difference at a target-error distance of $1.5^{\circ}[F(1,8)=11.88, p<.009]$, but not at $2.5^{\circ}$ $[F(1,9)=0.86]$ or $3.5^{\circ}[F(1,6)=0.87]$.

\section{Independence Model}

We can further ask whether the effects of these two bases for detection combine in an independent, additive manner suggested by Equation 1 (such a model specifies that the observer correctly detects a change in the object shift condition if the perceptual system perceives target object displacement, if it perceives changed spatial relations, or if the observer guesses correctly):

$$
f(d)=\eta(d)+\delta(d)+\text { Guess, }
$$

where $\eta(d)$ is the probability of perceiving target object displacement as a function of target-error distance, $d$; $\delta(d)$ is the probability of perceiving changed spatial relations as a function of target-error distance; and Guess, a constant, is .05 , the false alarm rate in the control condition. The likelihood of a correct response, $f(d)$, is between 0 and 1.

Expressing the likelihood of detection as $f(d)=1-$ (likelihood of no detection) and rearranging terms, we get

$$
f(d)=.05+[\delta(d)+\eta(d)-\delta(d) * \eta(d)] * .95 \text {. }
$$

Estimates for the unknowns in Equation 2 can be derived from actual observations in the all and background shift conditions by correcting the observations for guessing by using the transformations

$$
\begin{aligned}
& O_{\mathrm{ALL}}(d)=\eta(d)+[1-\eta(d)] * .05, \\
& O_{\mathrm{BKG}}(d)=\delta(d)+[1-\delta(d)] * .05,
\end{aligned}
$$

where $O_{\mathrm{ALL}}(d)$ is the observed likelihood of detection of an all shift at a given target-error distance, and $O_{\mathrm{BKG}}(d)$ is the observed likelihood of detection of a background shift at a given target-error distance.

A predicted curve for the object shift condition was derived by using Equation 3 to obtain estimates for $\eta(d)$ and $\delta(d)$ for each data bin for each subject, using Equation 2 to produce a prediction for the object shift condition and then averaging predictions across subjects. ${ }^{4}$ The resulting curve is shown in Figure 4. There was no significant difference between the observed and the predicted data points $[F(1,9)=2.99, F(1,9)=0.33$, and $F(1,7)=$ 1.43 for target error distances of $1.5^{\circ}, 2.5^{\circ}$, and $3.5^{\circ}$, respectively]. Thus, the data fail to reject the additive model.

In sum, then, the analysis of detection as a function of target-error distance supports the idea that there are two bases for detection in the object shift condition. Furthermore, as target-error distance increases, the likelihood of detecting target object displacement increases. Finally, given the assumptions made in the model, Figure 4 supports the hypothesis that these two bases combine in an independent manner to produce detection in the object shift condition.

\section{DISCUSSION}

Traditionally, visual stability across saccades has been accounted for by postulating an extra-retinal signal that globally remaps retinal space during each saccade. The saccade target theory (McConkie, 1991; McConkie \& Currie, 1996) provides an alternative account that does not assume an extra-retinal signal or an automatic, global remapping of retinal space. Instead, it postulates that local information is used in establishing a mapping function between the mental representation (developed from information obtained during past fixations) and retinal space (which can be used as needed to coordinate between these two domains).

The experiment reported here has demonstrated the relative importance of the saccade target object in detecting image displacements; detection is much higher when only that one object is displaced than when everything else in the image is displaced. Furthermore, detection of intrasaccadic displacements is a function of the direction of the displacement relative to the direction of the saccade (with or against), a finding that can be accounted for on the basis of the distance the eyes land from their target under the different conditions. The experiment provided evidence for the existence of two bases for detecting displacements, one being the retinal location of the 
saccade target object at the end of the saccade, and the other being a discrepancy between current and previously obtained information in the image, here represented as a difference in spatial relationships between the saccade target object and other, nearby objects.

In addition, there appears to be a time difference in when these are detected, as is proposed in the saccade target theory: Detection on the basis of retinal location occurs earliest, and detection on the basis of spatial relations occurs later. The theory assumes that the first of these is part of a process by which coordination of perception between eye fixations is achieved, with the second occurring only during perception that is enabled by this earlier process. The strengths of these two signals are both a function of eye position with respect to the saccade target object (e.g., the retinal location of that object), but the nature of these functions is quite different. Finally, the two signals appear to combine their effects on detection in the object shift condition in an independent manner.

These observations are all consonant with the saccade target theory and raise problems for an EEPI theory, particularly one that makes the assumption of a global retinal remapping. One way to revise an EEPI theory, to make it more compatible with these results, is to assume that local information in the region of the fovea has priority in evaluating whether the image is at the expected place on the retina. This is reasonable, given the fact that this information is being resolved with greater acuity. Although this can account for why the system is more sensitive to the displacement of objects lying in or near the fovea than for objects further away, it does not explain why shift direction relative to the saccade direction should have an effect. Moreover, people are more likely to detect displacements that result in the saccade target object's being placed further from the center of vision. This cannot easily be explained on the basis that the system is less sensitive to displacements at more peripheral locations. Furthermore, the stimuli used in this study were complex photographic images; there was no place without stimulus information, even if it was only texture.

Thus, it is not clear how a revised EEPI theory can account for the shift direction effect without including a mechanism that evaluates the distance of a particular part of the pattern (probably the object to which the eyes are being sent) with respect to the center of vision. This change would, of course, be an incorporation of essential characteristics of the saccade target theory itself.

At this point, it is reasonable to ask about any remaining differences between these two models. Clearly, the global remapping assumption is not a critical component of EEPI-based models, although traditionally it has been assumed. The critical difference between the two models is the use of EEPI. EEPI-based models make the hard assumption that the brain continually uses EEPI to maintain a space-constant representation of the visual world, in order to maintain a perception of stability. The basis for detecting intrasaccadic stimulus shifts is the resolution of this representation and the accuracy of the EEPI signal. For the detection of shifts, the saccade target theory, as formulated in the introduction, requires neither of these assumptions.

Although it is clear from many studies that the brain does encode EEPI, the critical question for the saccade target theory is whether it can explain the detection of shifts without an EEPI signal. If it can, this would mean that the saccade target theory is fundamentally different from other theories that explain the detection of stimulus shifts by reference to EEPI. However, if it cannot, EEPI-based theories and the saccade target theory may complement each other at some level. These questions must await further research.

Further questions concern the role of attention. For example, it is possible that signaling the saccade target object by wiggling it may have exogenously drawn attention to that object. This may have caused the participants to be more sensitive to changes in its location than would have been the case in ordinary viewing. This is a valid concern; however, McConkie and Currie (1996) did use a paradigm in which participants freely viewed scenes and still found indirect evidence that the system was detecting picture shifts based on a saccade target object.

Finally, although attention undoubtedly plays some role in any perceptual/cognitive process that occurs just before a saccade, the fact that detections were higher when the target shifted in the same direction as the saccade, as opposed to the opposite direction, indicates that attention alone is not sufficient to account for our findings. In other words, displacement detection did not depend solely on whether the target was attended, but on where the eyes landed, as the target-error distance analyses demonstrated.

In sum, by assuming that a fundamental perceptual goal of the system is to "find" a saccade target object within a limited retinal search region, the saccade target theory proposes that intrasaccadic stimulus shifts are detected because they disrupt feature-level, saccade target identification routines at the beginning of a new fixation. If appropriate visual information is available to support these identification routines, we perceive a stable world.

\section{REFERENCES}

Boyce, S., \& Pollatsek, A. (1992). Identification of objects in scenes: The role of scene background in object naming. Journal of Experimental Psychology: Learning, Memory, \& Cognition, 18, 531-543.

Bridgeman, B. (1981). Cognitive factors in subjective stabilization of the visual world. Acta Psychologica, 48, 111-121.

Bridgeman, B., Hendry, D., \& Stark, L. (1975). Failure to detect displacements of the visual world during saccadic eye movements. $\mathrm{Vi}$ sion Research, 15, 719-722.

Bridgeman, B., van der Heijden, A., \& VelichKovsky, B. (1994). A theory of visual stability across saccadic eye movements. Behavioral \& Brain Sciences, 17, 247-292.

Brune, F., \& Lücking, C. H. (1969). Okulomotorik, Bewegungswahrnehmung und Raumkonstanz der Sehdinge. Der Nervenarzt, 40, 692-700. 
Burr, D. C., Morrone, M. C., \& Ross, J. (1994). Selective suppression of the magnocellular visual pathway during saccadic eye movements. Nature, 371, 511-513.

Campbell, F. W., \& Wurtz, R. H. (1978). Saccadic omission: Why we do not see a gray-out during a saccadic eye movement. Vision Research, 18, 1297-1303.

Duhamel J.-R., Colby C. L., \& Goldberg, M. E. (1992). The updating of the representation of visual space in parietal cortex by intended eye movements. Science, 255, 90-92.

GRUSSER, O. J. (1972). Metacontrast and the perception of the visual world. European Journal of Physiology, 332(Suppl.), R98.

Haarmeier, T., Thier, P., Repnow, M., \& Petersen, D. (1997). False perception of motion in a patient who cannot compensate for eye movements. Nature, 389, 849-852.

Helmholtz, H. von (1963). Handbuch der physiologischen Optik [Handbook of physiological optics]. In J. P. C. Southall (Ed. and Trans.), Helmholtz's treatise on physiological optics (Vol. 3, pp. 247270). New York: Dover. (Originally published in 1866; English translation originally published in 1925)

Henderson, J. M. (1993). Eye movement control during visual object processing: Effects of initial fixation position and semantic constraint. Canadian Journal of Experimental Psychology, 47, 79-98.

IRWIN, D. E. (1992a). Memory for position and identity across eye movements. Journal of Experimental Psychology: Learning, Memory, \& Cognition, 18, 307-317.

IRWIN, D. E. (1992b). Perceiving an integrated visual world. In D. E. Meyer \& S. Kornblum (Eds.), Attention and performance XIV: Synergies in experimental psychology, artificial intelligence, and cognitive neuroscience (pp. 121-142). Cambridge, MA: MIT Press

Li, W., \& Matin, L. (1990). The influence of saccade length on the saccadic suppression of displacement detection. Perception \& Psychophysics, 48, 453-458.

MACK, A. (1970). An investigation of the relationship between eye and retinal image movement in the perception of movement. Perception \& Psychophysics, 8, 291-298.

MaCkNik, S. L., Fisher, B. D., \& Bridgeman, B. (1991). Flicker distorts visual space constancy. Vision Research, 31, 2057-2064.

Matin, L. (1986). Visual localization and eye movements. In K. R. Boff, L. Kaufman, \& J. P. Thomas (Eds.), Handbook of perception and human performance (Vol.1, pp. 20.1-20.45). New York: Wiley.

MCConkie, G. M. (1991, September). Perceiving a stable visual world. Paper presented at the 6th European Conference on Eye Movements, Leuven, Belgium.

McConkie, G. W., \& Currie, C. (1996). Visual stability across saccades while viewing complex pictures. Journal of Experimental $P$ sychology: Human Perception \& Performance, 22, 563-581.
O'Regan, J. K. (1992). Solving the "real" mysteries of visual perception: The world as an outside memory. Canadian Journal of Psychology, 46, 461-488.

SkAVENSKI, A. (1990). Eye movements and visual localization of objects in space. In $\mathrm{E}$. Kowler (Ed.), Eye movements and their role in visual and cognitive processes (pp. 263-287). New York: Elsevier.

Volkmann, F. C., Schick, A. M., \& Riggs, L. A. (1968). Time course of visual inhibition during voluntary saccades. Journal of the Optical Society of America, 58, 562-569.

von Holst, E., \& Mittelstaedt, H. (1971). The principle of reafference: Interactions between the central nervous system and the peripheral organs. In P. C. Dodwell (Ed. \& Trans.), Perceptual processing: Stimulus equivalence and pattern recognition (pp. 41-71). New York: Appleton. (Original work published 1950)

Whipple, W. R., \& Wallach, H. (1978). Direction-specific motion thresholds for abnormal image shifts during saccadic eye movement, Perception \& Psychophysics, 24, 349-355.

\section{NOTES}

1. We are not assuming any specific retinal-based neuromechanism. By the term retinal, we mean the perceptual system's selective use of information from the retinal array, as opposed to the perceptual system's use of extra-retinal information.

2. We were not able to conduct this analysis with trials in which the picture was seen only for the first time, because of insufficient data. Nevertheless, the means in this case followed the same pattern as the means shown in Figure 2: Detection likelihoods for the object shift, all shift, background shift, and control conditions were $.83, .50, .30$, and .05 respectively.

3. To make a same versus opposite classification, each saccade was represented as a vector, decomposing the direction of the saccade into a horizontal $x$-component and a vertical $y$-component. Whichever component was largest (the $x$-or the $y$-component) defined the primary saccade component. Classification occurred by whether the primary saccade component was in the same or the opposite direction as the picture shift. When the star (or initial starting point of the eyes) was at $0^{\circ}, 20^{\circ}$, or $40^{\circ}$, the primary saccade component was generally horizontal; at $60^{\circ}$, it was generally vertical.

4. The predicted object shift curve used actual background shift data from the $0.5^{\circ}, 1.5^{\circ}$ bins. To derive predictions for target-error distances greater than $1.5^{\circ}$, the model simply assumed that any detection of changed spatial relations would remain constant at $37 \%$.

(Manuscript received March 1, 1998; revision accepted for publication May 18, 1999.) 